\title{
Novel Strategies for the Management of Recurrent Pregnancy Loss
}

\author{
William H. Kutteh, MD, PhD, HCLD ${ }^{1,2}$ \\ ${ }^{1}$ Department of Obstetrics and Gynecology, Vanderbilt University \\ Medical Center, Nashville, Tennessee \\ 2 St. Jude Children's Hospital, Memphis, Tennessee \\ Address for correspondence William H. Kutteh, MD, PhD, HCLD, \\ Director, Recurrent Pregnancy Loss Center, Fertility Associates of \\ Memphis, 80 Humphreys Center, Suite 307, Memphis, TN 38120 \\ (e-mail: wkutteh@fertilitymemphis.com).
} Semin Reprod Med 2015;33:161-168

\begin{abstract}
Keywords

- recurrent pregnancy loss

- antiphospholipid antibodies

- uterine anomalies

- recurrent miscarriage

This article discusses the current trends in the diagnosis and treatment of recurrent pregnancy loss. Genetic testing of the miscarriage tissue by 23-chromosome microarray and the ability to identify maternal cell contamination have increased our awareness of the role of aneuploidy as a cause of recurrent pregnancy loss. This increasing influence and the role of genetic testing in developing a strategy for the evaluation of recurrent pregnancy loss are described and discussed. The most common questions that practicing physicians ask about recurrent pregnancy loss include how many losses are needed to make the diagnosis, what counts as a pregnancy loss, what constitutes a full workup, should we get karyotypes on the parents and the miscarriage, and what is the prognosis for a live birth? This review attempts to answer those questions based on current research and clinical experience to expand our current understanding of recurrent pregnancy loss.
\end{abstract}

There are several controversial topics in the diagnosis and management of recurrent pregnancy loss (RPL). This review discusses some of these topics as well as the current trends in the diagnosis and treatment of RPL. The different definitions of the governing societies of "pregnancy" and of "recurrent pregnancy loss" are described. The increasing influence and the role of genetic testing in developing a strategy for the evaluation of RPL are reviewed. Finally, the effect of maternal age and the number of prior losses on predicting future outcome of pregnancy in couples with RPL must be appreciated.

As director of the Recurrent Pregnancy Loss Center in Memphis, we are contacted by outside physicians who have questions about one of their patients with RPL. These questions usually fall into one of the common areas listed in - Table 1. This review attempts to answer those questions based on our current understanding of RPL.

\section{Natural Miscarriage History}

During my fellowship and subsequent faculty years at Parkland Hospital in Dallas, I was able to calculate the recurrent miscarriage history from the large patient population. In the early 1990s, there were approximately 15,000 deliveries per year at Parkland Hospital. Using a computerized search method of patients who presented to Parkland Gynecology and Obstetrical Clinics, a frequency of RPL was determined to be $1.3 \%$ based on at least 766 RPL cases out of 59,035 deliveries. It is important to note that at that time RPL was defined as three or more consecutive losses, so this would follow strict criteria. Moreover, this population was neither selected nor referred and consisted of approximately equal proportions of Caucasian, Hispanic, African American ethnicities. This estimate of $1.3 \%$ as the frequency of RPL should be considered as a minimum because of these factors. ${ }^{1}$

Other individual investigators have calculated a theoretical incidence of miscarriage based on the number of miscarriage used to define RPL. ${ }^{2}$ For example, if two miscarriages are the defining number, the theoretical incidence of miscarriage is 1 in 45 women. If four miscarriages are used to define RPL, the incidence is 1 in 2,000. If six miscarriages are used to define RPL, the incidence is 1 in 90,000.
Issue Theme Innovations in Reproductive Endocrinology: A Tribute to Bruce Carr, MD; Guest Editors, Serdar E. Bulun, MD and Richard S. Legro, MD
Copyright (c) 2015 by Thieme Medical Publishers, Inc., 333 Seventh Avenue, New York, NY 10001, USA. Tel: +1(212) 584-4662. 
Table 1 Common questions about RPL

\begin{tabular}{|l|}
\hline 1. How many losses are required to diagnose RPL? \\
\hline 2. What counts as a pregnancy loss? \\
\hline 3. What constitutes a full workup of RPL? \\
\hline 4. Should we get karyotypes on both parents? \\
\hline 5. Should we get a genetic analysis on the miscarriage? \\
\hline 6. What is the prognosis for a live birth? \\
\hline
\end{tabular}

Abbreviation: RPL, recurrent pregnancy loss.

It is also important to consider the role of maternal age when considering RPL. For example, the theoretical incidence of sporadic miscarriages based on maternal age has been calculated using the following formula: Incidence $=\mathrm{u}^{2}$ ( $u$ equals sporadic miscarriage rate for age). ${ }^{2}$ In this theoretical calculation, maternal age of 20 would correlate with an incidence of 1 in 85 for RPL. By age 30, the incidence would rise to 1 in 45 women of reproductive age, and by age 40 it would rise to a theoretical incidence of RPL of 1 in 4 . It is also well known that the incidence of spontaneous miscarriage increases in relation to maternal age. For example, in women age 25 to 29 the frequency of spontaneous miscarriage is approximately 10 to $15 \%$. By age 35 to 39 , this rate of spontaneous miscarriage increases to 25 to $30 \%$ and by age 40 to 44 , this has risen to 50 to $55 \%{ }^{3}$

The relationship of maternal age to miscarriage correlates with the frequency of aneuploidy in oocytes. Based on data from chromosomal abnormalities detected in in vitro human oocytes, the frequency of aneuploidy was determined based on maternal age. ${ }^{4}$ In women younger than 35 , aneuploidy in the oocytes was $10 \%$ (-Table 2). By age 43 the frequency of aneuploidy in oocytes increased to $50 \%$ and in those women over 45 , virtually $100 \%$ of oocytes demonstrated some aneuploidy.

\section{Number of Losses Required to Define Recurrent Pregnancy Loss}

As discussed previously, the theoretical incidence of RPL depends on the number of losses required to meet the definition. Traditionally, RPL was defined as three or more spontaneous consecutive pregnancy losses, and in some cases also included a statement that the same partner fathered them. In 2001, the American College of Obstetricians and Gynecologists (ACOG) defined RPL as "two or three or more

Table 2 Maternal age related to aneuploidy in oocytes

\begin{tabular}{|l|l|}
\hline Aneuploidy risk (\%) & Maternal age (y) \\
\hline 10 & $<35$ \\
\hline 30 & 40 \\
\hline 50 & 43 \\
\hline 100 & 45 \\
\hline
\end{tabular}

Note: Percentage of human oocytes that had aneuploidy based on maternal age. consecutive pregnancy losses," but this bulletin has been withdrawn and has not been updated at the time of this writing. ${ }^{5}$ In 2012, the American Society for Reproductive Medicine Practice Committee issued a statement that defined RPL "as a disease distinct from infertility defined by two or more failed consecutive pregnancies." 6 The Royal College of Obstetricians and Gynaecologists in their Green top Guidelines defines recurrent miscarriage as "the loss of three or more consecutive pregnancies."7 Based on my experience over the past 20 years in clinical practice, most insurance companies will agree to pay for complete evaluation for RPL after two consecutive losses.

There are still some physicians that feel RPL is not really a clinical disease but rather is an unfortunate occurrence by chance in some women. Though this may be true in some cases, the theoretical incidence of miscarriage occurring by chance for women with one, two, and three miscarriages has been calculated. ${ }^{2}$ For example, in women age 20 to 24 , the incidence of miscarriage due to chance is calculated to be $11 \%$, whereas after two miscarriages this drops to $1.2 \%$, and after three miscarriages it drops to $0.13 \%$. For a woman age 30 to 34 , the chance of one miscarriage occurring by chance is theoretically $15 \%$, whereas two miscarriages occurring by chance in this age group would be $2.25 \%$ of couples. Once women had three miscarriages at age 30 to 34 , the theoretical incidence of RPL by chance alone would be $0.34 \%$. These theoretical numbers for estimating the frequency of RPL by chance alone, particularly for three miscarriages by chance alone, are significantly lower than the numbers that were determined in our minimal estimate of the frequency of RPL based on almost 60,000 women evaluated at Parkland Hospital. ${ }^{1}$ Thus, it can be concluded that there are other factors, in addition to chance, that are contributing to recurrent miscarriage.

We published one of the largest studies ever reported on the frequency of abnormal tests in more than 1,000 women with RPL. ${ }^{8}$ In this report, we categorized women based on the number of losses that they had experienced and grouped them as those that had only two losses, only three losses or four or more losses. We developed a list of "evidence-based tests" based on the current literature that included karyotypes on the parents, uterine anatomy evaluation, testing for lupus anticoagulant and anticardiolipin antibodies, and tests for thyroid-stimulating hormone and factor $\mathrm{V}$ Leiden DNA. ${ }^{9,10}$ We also included "investigative tests" that were under study at that time, including antiphosphatidylserine antibodies, mid-luteal progesterone levels, cervical cultures for Mycoplasma spp. and Ureaplasma spp., factor II (prothrombin) gene mutation, and prolactin. When we tabulated the findings based on the number of prior losses (-Table 3 ), there were no differences in the evidence-based test results or investigative test results based on number of prior losses (-Table 3) of two, three, or more than four. ${ }^{8}$ These data strongly argued that an evaluation for pregnancy loss should be initiated after two losses. This recommendation was confirmed by the ASRM Committee opinion that stated, "clinical evaluation may proceed following two first trimester pregnancy losses." 6 
Table 3 Recurrent pregnancy loss etiologies based on number of prior losses

\begin{tabular}{|l|l|l|l|l|}
\hline & $\begin{array}{l}\mathbf{2} \text { prior losses } \\
(\boldsymbol{n}=\mathbf{4 4 7})\end{array}$ & $\begin{array}{l}\mathbf{3} \text { prior losses } \\
(\boldsymbol{n}=\mathbf{3 4 3 )}\end{array}$ & $\begin{array}{l}\geq \mathbf{4} \text { prior losses } \\
(\boldsymbol{n}=\mathbf{2 3 0})\end{array}$ & $\begin{array}{l}\boldsymbol{p} \text { value } \\
(\mathbf{2} \text { vs. 3 vs. }>\mathbf{3})\end{array}$ \\
\hline Evidence-based test results & $41 \%$ & $40 \%$ & $42 \%$ & NS \\
\hline Investigative test results & $20 \%$ & $22 \%$ & $21 \%$ & NS \\
\hline Total abnormal test results & $61 \%$ & $62 \%$ & $63 \%$ & NS \\
\hline
\end{tabular}

Notes: Frequency of abnormal test results in 1,020 women with recurrent pregnancy loss. See text for details about "Evidence-based tests" and "Investigative tests."

Source: Data modified from Jaslow et al. ${ }^{8}$

\section{What Counts as a Pregnancy Loss}

Certainly, the definition of a pregnancy will affect the definition of a pregnancy loss. There is a spectrum for pregnancy loss based on gestational age that has been outlined in a recent publication. ${ }^{11}$ This categorization of pregnancy losses includes pregnancies of unknown location and goes all the way through miscarriages, which are less than 20 weeks (-Table 4). The traditional definition of a pregnancy loss came from Williams Obstetrics textbook that stated that "a miscarriage is the loss of a pregnancy before 20 weeks of gestation or less than 500 grams." 12 This definition made diagnosis of a pregnancy loss very easy; any woman who had a confirmed pregnancy that failed was categorized as having a miscarriage.

ACOG defined a miscarriage as "loss of a recognized pregnancy in the first or early second trimester at less than 15 gestational weeks." ${ }^{5}$ The Practice Bulletin, which has been withdrawn, went on to state that most pregnancy losses "are evident by the 12 th week and the demise precedes the clinical features of pregnancy loss by one or more weeks." This latter statement is clearly true as all clinicians know, and patients ultimately learn that the demise of a pregnancy often precedes any outward signs of loss by several weeks. This fact complicates some of the definitions that have been suggested that define certain stages of a loss based gestational weeks. ${ }^{11}$ We have found at the Recurrent Pregnancy Loss Center that it is most useful to provide the information concerning last menstrual period and detailed information about what if anything was seen on ultrasound. Particular attention should be made to the gestational sac, the yolk sac, the fetal pole, and the presence or absence of fetal heart motion. To define pregnancy loss, ASRM Practice Committee first defined pregnancy "as a clinical pregnancy documented by ultrasonogra-

Table 4 Spectrum of pregnancy loss

\begin{tabular}{|l|}
\hline - Pregnancy of unknown location (PUL) \\
\hline - Early embryonic Loss (<6 wk) \\
\hline - Embryonic loss ( $>6-9 \mathrm{wk})$ \\
\hline - Fetal loss ( $>9-20 \mathrm{wk})$ \\
\hline - Miscarriage $(<20 \mathrm{wk})$ \\
\hline - Stillbirth (>20 wk) \\
\hline
\end{tabular}

Source: Nomenclature from Silver et al. ${ }^{11}$ phy or histopathologic examination."6 This Practice Bulletin has added some confusion to the field in that a pregnancy that is lost without an ultrasound being performed or without the products of conception being evaluated does not "count" as a miscarriage. It further does not consider those well-documented pregnancies that are termed "biochemical losses."

The Royal College of Obstetrics and Gynecology defined miscarriage as "a spontaneous loss of pregnancy before the fetus reaches viability." 7 Their guideline states "all pregnancies losses from the time of conception until 24 weeks of gestation should be included." This more inclusive definition seems more practical based on clinical experience. Certainly, many patients consider a miscarriage any pregnancy in which they have had a positive pregnancy test that does not result in the birth of baby. In our large studies, we have followed the definition of a pregnancy loss as a pregnancy that "is documented by an appropriately rising quantitative hCG that ultimately fails." 8 Using this definition, the chance of error in defining an intrauterine pregnancy loss as an ectopic pregnancy is less than $7 \%{ }^{13}$

\section{What Is a Complete Workup}

When I became a faculty member at UT Southwestern Medical Center in 1991, the workup of RPL was fragmented. If a patient was referred to the Maternal Fetal Medicine Clinic, the patient and her partner had a very detailed pedigree and chromosome testing was performed. Detailed counseling about other possible causes was always included. Patients' referred to Gynecology Clinic or Reproductive Endocrinology Clinic with RPL had a variety of tests performed. The inconsistent testing sometimes included a hysterosalpingogram, anticardiolipin antibodies, and some hormonal tests. In checking the literature and discussing RPL with my colleagues around the country, I found that these differences were not just at UT Southwestern but were found in other clinical locations.

As interest in patients with RPL increased, it became important to gather data from patients with RPL in a centralized location. To follow the "Parkland Way" I dutifully went from office to office of most of the faculty at Parkland to ask whether "it was all right if I saw the RPL patients in my clinic." Almost everyone gladly agreed to let me see these patients as they presented a clinical challenge. As a result of this action, I was able to see more than 2,000 patients with RPL during my 7 years in Dallas. 
The ongoing challenge to the concerned physician is to determine the appropriate tests to complete once a patient with recurrent miscarriage has been identified. In other words, they have considered both the number of losses and what counts as a loss and a patient with RPL now sits in their office and desires testing, treatment, and answers. Based on the ACOG Practice Bulletin that was written in 2001 and subsequently withdrawn, the complete evaluation for RPL included karyotypes on both partners, a uterine cavity evaluation, a glucose level, a lupus anticoagulant test, and testing for anticardiolipin antibodies. ${ }^{6}$ A recent ACOG Practice Bulletin on antiphospholipid syndrome indicates that $\beta$ II glycoprotein I antibodies should also be included in the evaluation. ${ }^{14}$ After many years of controversy and multiple Practice Bulletins, the most recent recommendation from ACOG indicates that testing for thrombophilia in women with RPL is not indicated unless there is a personal history of thrombosis and/or a strong family history of thrombosis. ${ }^{15}$ The recent Committee Opinion of the American Society of Reproductive Medicine (ASRM) states that karyotypes on both partners should be obtained, and a careful uterine cavity evaluation, tests for lupus anticoagulant, anticardiolipin antibodies, and anti- $\beta$ II glycoprotein I antibodies should be obtained, in addition to tests for progesterone, polycystic ovarian syndrome, and hemoglobin $\mathrm{A}_{1 \mathrm{c}}{ }^{6}$. This panel of testing at present should be considered as a complete evaluation under current guidelines. ASRM Committee Opinion, in agreement with the ACOG Practice Bulletin, does not indicate the need for testing for inherited thrombophilia in the absence of a personal history of thrombosis and/or a strong family history of thrombosis.

\section{Genetic Testing on the Parents}

It is often argued that genetic testing on the parents is not necessary because the frequency of genetic abnormalities in the parents from couples with RPL is only 3 to $5 \%{ }^{8}$ The second most commonly quoted reason is that genetic testing with karyotype analysis is expensive. Conventional karyotypes cost approximately $\$ 850$ each with 23-chromosome microarray being more expensive. The frequency of genetic abnormalities in the parents was carefully described in our paper based on the number of losses two, three, or four or more ( - Table 5). In couples with RPL who have a parental genetic abnormality, a balanced translocation is the genetic abnormality in $85 \%$ of cases. ${ }^{16}$ These include both reciprocal translocations (59\%) and Robertsonian translocations (27\%). Inversions (9\%), sex chromosome aneuploidies (4\%), and supernumerary chromosomes (1\%) are detected less frequently. The importance of obtaining karyotypes on the parents should not be minimized. The karyotype results from the parents can provide prognostic information for subsequent pregnancies. For example, if the parent has a reciprocal translocation, the chance of a subsequent miscarriage is 50 to $70 \%$. If one the parent harbors a Robertsonian translocation, the chance for a subsequent miscarriage is 30 to $50 \%$ with the exception being if the translocation is to the same chromosome. Similarly, inversions in parental chromosomes result in subsequent miscarriages of at least $30 \%{ }^{16-21}$

\section{Genetic Evaluation of the Products of Conception}

The karyotype obtained from the miscarriage tissue or products of conception also is useful for providing prognosis for subsequent pregnancy outcome. For example, if the products of conception from the first miscarriage are normal, the chance that the second miscarriage will have a normal karyotype is $65 \%{ }^{22-24}$ Conversely, if the genetic analysis on the products of conception from the first miscarriage is aneuploid, the second miscarriage will be aneuploid in $65 \%$ of cases. The risk of aneuploidy has been studied in women with sporadic miscarriages compared with women with recurrent miscarriages. ${ }^{25}$ Interestingly, the risk of cytogenetic abnormalities in the products of conception from women with RPL is lower in every age group studied than the frequency of genetic abnormalities in the products of conception from women who had sporadic miscarriages. For example, the frequency of cytogenetic abnormalities in the miscarriage tissue from women younger than 35 who had sporadic pregnancy loss was approximately $50 \%$. However, cytogenetic abnormalities in the miscarriage tissue from women with RPL were considerably lower at approximately

Table 5 Etiologies of evidence-based tests when evaluating recurrent pregnancy loss

\begin{tabular}{|l|l|l|l|l|l|}
\hline $\begin{array}{l}\text { Control } \\
\text { Frequency (\%) }\end{array}$ & Abnormal test result & $\begin{array}{l}\mathbf{2} \text { prior losses } \\
(\boldsymbol{n}=\mathbf{4 4 7})\end{array}$ & $\begin{array}{l}\mathbf{3} \text { prior losses } \\
(\boldsymbol{n}=\mathbf{3 4 3})\end{array}$ & $\begin{array}{l}\geq \mathbf{4} \text { prior losses } \\
(\boldsymbol{n}=\mathbf{2 3 0})\end{array}$ & $\begin{array}{l}\boldsymbol{p} \text { value } \\
(\mathbf{2} \text { vs. 3 vs. }>\mathbf{3})\end{array}$ \\
\hline 0.4 & Parental genetics & $2.8 \%$ & $5.4 \%$ & $5.2 \%$ & NS \\
\hline 7.5 & Uterine anatomy & $18.7 \%$ & $18.2 \%$ & $16.7 \%$ & NS \\
\hline 0.5 & Lupus anticoagulant & $5.0 \%$ & $2.9 \%$ & $1.9 \%$ & NS \\
\hline 6.7 & Anticardiolipin antibodies & $15.6 \%$ & $13.1 \%$ & $17.1 \%$ & NS \\
\hline 3.9 & Thyroid-stimulating hormone & $8.1 \%$ & $6.5 \%$ & $6.2 \%$ & NS \\
\hline 6.8 & Factor V Leiden mutation & $4.2 \%$ & $8.1 \%$ & $10.3 \%$ & NS \\
\hline
\end{tabular}

Notes: Frequency of abnormal test results in 1,020 women with recurrent pregnancy loss. Control frequency based on the percent of reproductiveaged, nonpregnant women without a history of miscarriage who had an abnormal test result.

Source: Data modified from Jaslow et al. ${ }^{8}$ 
$35 \%$. This increased frequency of cytogenetic abnormalities in women with sporadic versus recurrent miscarriage persisted even in women older than 40 (70 vs. $62 \%$, respectively). ${ }^{25}$ These data strongly argue that genetic factors alone cannot be the causative factor for miscarriages in many women with recurrent miscarriage.

Studies on preimplantation embryos from women with RPL undergoing in vitro fertilization provide interesting data when compared with the genetic abnormalities found in the miscarriage tissue. ${ }^{26}$ The possibility exists that certain aneuploidies and other abnormalities that are detected in miscarriage tissue from women with RPL represent those abnormalities that restricted the pregnancy grow up to a certain point. The most common chromosomal abnormalities seen in products of conception include those from chromosome $16,22,21$, and 15 . These account for almost $60 \%$ of all genetic results. Conversely, aneuploidies in chromosome 1 are rarely seen in products of conception, whereas those from chromosomes 2 through 7 are seen less than $10 \%$ of the time. Recent data from preimplantation genetic screening on more than 2,000 embryos from RPL patients who underwent in vitro fertilization indicate that aneuploidy in the developing embryo exists at significant rates in all 23 pairs of chromosomes when evaluated both at the cleavage stage and the blastocysts stage, as identified by microarray preimplantation genetic screening on couples with two or more prior pregnancy losses. ${ }^{27,28}$ For example, the frequency of both monosomy and trisomy in all chromosomes was fairly equally distributed with the aneuploidy rate ranging from 3.1 to $5.8 \%$. This is in stark contrast to the aneuploidy rates that are seen in the miscarriage tissue in which abnormalities in chromosomes 15, 16, 21, and 22 account for $60 \%$ of the abnormalities that are seen. This strongly suggests that many of the pregnancy losses that we term "biochemical" are due to aneuploidies in some of the larger chromosomes and that there is such a disparity in genetic material that is present in the embryo that growth cannot continue more than a few days before the pregnancy fails.

\section{Anatomical Causes of Recurrent Pregnancy Loss}

Both congenital and acquired uterine anomalies are felt to contribute to RPL. ${ }^{29}$ We recently reported on the prevalence of uterine anomalies in more than 900 consecutive patients with recurrent miscarriage. ${ }^{30}$ Based on this study, the frequency of significant uterine anomalies was $19.5 \%$ with congenital anomalies accounting for $6.2 \%$ and acquired anomalies accounting for $13.3 \%$. As shown in - Table 6, septate uterus is the most common congenital anomaly with approximately $4.8 \%$ of women having this identified. Uterine fibroids were the most common acquired anomaly. Interestingly, a septate uterus was found significantly more frequently in women with primary recurrent miscarriage when compared with secondary recurrent miscarriage (6.3 vs. 3.1\%, $p=0.028$ ). The evaluation of uterine anomalies is easily detected in the office with the use of three-dimensional (3D) sonohysterography. The addition of saline infusion with 3D sonohysterography clearly identifies almost all uterine anomalies with the exception in the case of a unicornuate uterus with a nonconnecting horn. We use this technology almost exclusively at the Center for Recurrent Pregnancy Loss and rarely resort to other imaging modalities. Minimally invasive surgery is generally used to correct the uterine septum as well as for the removal of fibroids, adhesions, and polyps. ${ }^{31}$

\section{Immune Causes for Recurrent Pregnancy Loss}

Both autoimmune (immune reaction against self) and alloimmune (immune reaction against another) causes have been implicated as potential factors in RPL. Immune tolerance of

Table 6 Comparison of congenital and acquired uterine anomalies identified in women (primary vs. secondary RPL)

\begin{tabular}{|l|l|l|l|}
\hline & Primary RPL $(\boldsymbol{n}=479)$ & Secondary RPL $(\boldsymbol{n}=425)$ & $\boldsymbol{p}$ value \\
\hline All uterine anomalies & $22.8(109)$ & $15.8(67)$ & 0.009 \\
\hline Congenital anomalies & $8.8(42)$ & $4.5(19)$ & 0.011 \\
\hline Bicornuate uterus & $1.0(5)$ & $0.5(2)$ & NS \\
\hline Didelphic uterus & $0.2(1)$ & $0.2(1)$ & NS \\
\hline Septate uterus & $6.3(30)$ & $3.1(13)$ & 0.028 \\
\hline Unicornuate uterus & $0.8(4)$ & $0.5(2)$ & NS \\
\hline Acquired anomalies & $14.6(71)$ & $11.7(50)$ & NS \\
\hline Adhesions & $4.0(19)$ & $4.2(18)$ & NS \\
\hline Fibroids & $7.3(35)$ & $5.4(23)$ & NS \\
\hline Polyps & $4.0(19)$ & $2.4(10)$ & NS \\
\hline
\end{tabular}

Abbreviation: RPL, recurrent pregnancy loss.

Notes: Values represent \% occurrence with number of cases in parentheses. Primary recurrent pregnancy loss means that there had never been a live birth. Secondary recurrent pregnancy loss means that a series of losses followed a live birth.

Source: Modified from Jaslow and Kutteh. ${ }^{30}$ 
the developing fetus remains one of the challenging concepts for the field of immunology. Theoretically, the fetus should be rejected as an allograft. Clearly, this is not the case and the mechanisms behind the tolerance of or the failure to reject the fetal allograft remain to be elucidated. In the absence of a clear scientific explanation, several theories have been proposed to explain the breakdown of immunological tolerance of the developing fetus leading to RPL including similarities in human leukocyte antigens (HLA), the generation of the embryotoxic factors, the presence of natural killer cells, among others. But to date, none of these theories has clearly been shown to be true. ${ }^{32}$ Despite this lack of evidence, several tests have been developed, including embryotoxic factors, natural killer cell assays, T-helper cell 1 and 2 cytokine ratios, that have been proposed to help in the evaluation of RPL. Unfortunately, none of these tests has clearly been shown to differentiate the population of women with RPL from appropriate control groups, thus testing and ultimately treating these women at this time is not advised based on the recent Cochran review. ${ }^{33}$ The use of white blood cell immunotherapy, intravenous $\gamma$-globulin, and more recently intralipids remains both controversial and experimental, and requires further research. ${ }^{34}$

Conversely, autoimmune factors including the well-characterized antiphospholipid antibody syndrome should be evaluated in all women with RPL and are identified in 15 to $20 \%$ of women with RPL. ${ }^{35}$ The antiphospholipid antibody syndrome was the ideal clinical problem for me to focus on as it combined my interests in reproductive medicine and my $\mathrm{PhD}$ in molecular cell biology and immunology. Testing of all women for lupus anticoagulant, anticardiolipin antibodies and, more recently, testing for $\beta$ II glycoprotein I antibodies have been advised. ${ }^{14}$ Current clinical studies indicate that testing for antiphosphatidylserine may also be a significant clinical factor; however, this recommendation has not been advanced in any of the guidelines to date.

Early reports of women with antiphospholipid antibody syndrome included a few patients who had significant placental thrombosis. Based on this observation and the finding that the antiphospholipid antibody syndrome was present in some women with systemic lupus erythematosus, the association with thrombosis was established. However, both in vivo and in vitro studies on the pathophysiology of antiphospholipid antibodies indicate that much of what we understand about the action of these antibodies in RPL is not just on the coagulation pathway. ${ }^{36}$ For example, recent studies indicate that antiphospholipid antibodies inhibit the release of human chorionic gonadotropin (hCG) hormone from placental explants. Importantly, these antibodies also block the in vitro trophoblast migration and invasion as well as formation of the giant multinucleated syncytiotrophoblasts. Molecular studies have shown that antiphospholipid antibodies inhibit the trophoblast cell adhesion molecules $\left(\alpha_{1}\right.$ and $\alpha_{5}$ integrins as well as E and VE cadherins). Furthermore, antiphospholipid antibodies activate complement on the trophoblast surface inducing an inflammatory response. ${ }^{37}$ Clearly, we do not understand everything that is going on with antiphospholipid antibodies, but it is not just anticoagulation. The current therapy for antiphospholipid syndrome includes the use of heparin, ${ }^{38}$ which is known to reverse the inhibition of syncytiotrophoblasts formation as well as increase the production of hCG from placental explants in vitro. This mainstay of therapy has been shown in several studies to significantly increase the live birth rate. ${ }^{14}$

\section{Unexplained Recurrent Pregnancy Loss}

Despite a thorough evaluation for RPL, not all couples will have answers to their dilemma. When using evidence-based tests for evaluation of couples with RPL, approximately $40 \%$ of couples will have an etiology identified that could be associated with their loss. When additional investigative tests were added, this would contribute potentially another $20 \%$ of patients who might be provided an explanation for their loss. ${ }^{8}$ With the increased use of 23-chromosome microarray evaluation on miscarriage tissue, additional answers for couples are available. It has been estimated in several studies that approximately $50 \%$ of miscarriages from women of all ages with RPL can be explained by cytogenetic abnormalities in the pregnancy itself. ${ }^{16}$ Thus with a combination of evaluation of products of conception and careful evaluation of the genetic, anatomic, endocrine, and autoimmune status of the parents, the majority of couples can be provided with an explanation for their loss. A new testing evaluation algorithm has been established based on the testing of the products of conception after a second miscarriage (-Fig. 1). Using this algorithm, the karyotype on the second miscarriage determines the next step in the evaluation for RPL. If the miscarriage tissue was aneuploid, the explanation for the pregnancy loss was known and in the absence of other factors, no further evaluation would be necessary at that point in time. In those cases where the karyotype has not been obtained or a karyotype is normal, a full RPL evaluation should be performed. ${ }^{29}$

This still leaves a group of couples with truly unexplained RPL. Unexplained RPL is diagnosed after a complete evaluation of the genetic, anatomic, endocrine, and autoimmune factors have been completed on the couple and all of these have returned as normal. Second, the chromosomes on the products of conception have repeatedly returned as normal. Third, other factors such as lifestyle factors that we know contribute to miscarriage have been corrected or eliminated. These include tobacco use, alcohol consumption, and maternal obesity, all of which have been shown in multiple studies to significantly increase the risk of miscarriage. ${ }^{29}$ Finally, maternal age must be taken into consideration when classifying someone as unexplained RPL. When these criteria are used, it is a small group of women who truly are classified as unexplained. Encouraging data continue to be presented about subsequent chance of live birth in couples with $\mathrm{RPL}^{39}$ In the large, recent report, they evaluated the chance of the live birth in couples with RPL based both on the number of prior losses and maternal age. In this study, the initial time point began when the patient was referred for evaluation for RPL, all of the current testing and treatment was completed, and they had follow-up of 20 or more years on many of the 


\section{Initial Evaluation for Early RPL}

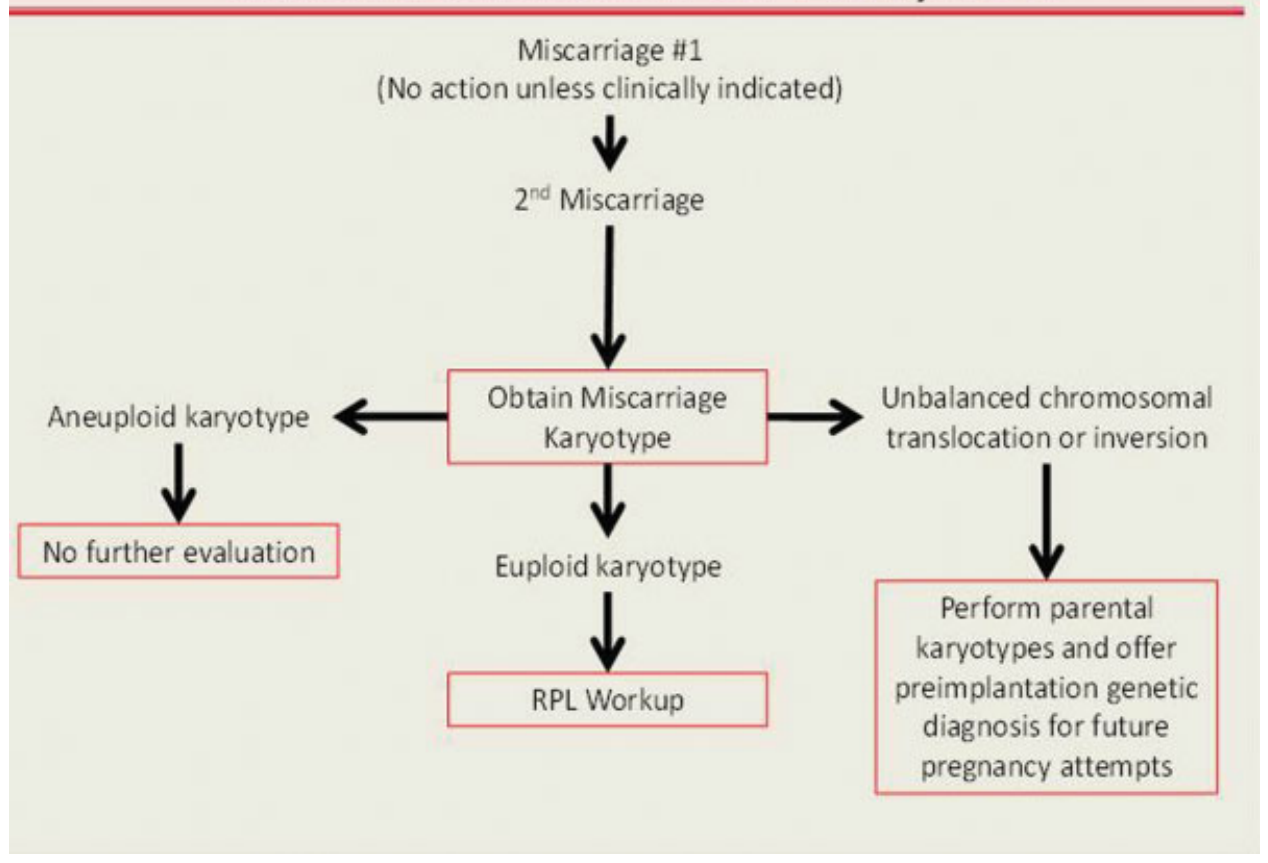

Fig. 1 Algorithm for the evaluation of recurrent pregnancy loss (RPL).

patients. For example, in women with three miscarriages, the chance of a successful live birth after about 2 years was $70 \%$, whereas, women with six or more miscarriages have only a $45 \%$ chance of a live birth after 2 years based on their data. When evaluating outcome based on maternal age, those women younger than 30 have approximately a $75 \%$ chance of live birth within 2 years, whereas those age 40 have a chance of live birth closer to $40 \%$. Thus, it is possible, after a thorough evaluation on the parents, an evaluation of the products of conception, evaluation of lifestyle factors, and considering maternal age and number of losses, to provide your couple with a realistic expectation of their chance of live birth in the future.

\section{Summary}

Recent advances in genetic testing on the products of conception are leading to a reorientation in our thought process for evaluating and treating women with RPL. Currently, many clinicians are obtaining 23-chromosome microarray test after the second miscarriage, and using this information to guide further evaluation. If the miscarriage karyotype is abnormal, and there are no other obvious factors, such as a submucosal fibroid that would contribute to pregnancy loss, no other testing need be performed initially. However, if the miscarriage karyotype is normal, or if the karyotype on the products of conception have not been obtained, or if subsequent miscarriages occur, it is indicated to move forward with a thorough evaluation including genetic evaluation of the parents, careful evaluation of the uterine cavity, hormonal assessment, and auto- immune testing. Based on the outcome of these tests, couples can be guided to the appropriate path for treatment and a realistic expectation for a successful outcome can be provided.

\section{References}

1 Kutteh WH, Pasquarette MM. Recurrent pregnancy loss. Adv Obstet Gynecol 1995;2:147-177

2 Saravelos SH, Regan L. Unexplained recurrent pregnancy loss. Obstet Gynecol Clin North Am 2014;41(1):157-166

3 Hassold T, Chen N, Funkhouser J, et al. A cytogenetic study of 1000 spontaneous abortions. Ann Hum Genet 1980;44(Pt 2):151-178

4 Pellestor F, Andréo B, Arnal F, Humeau C, Demaille J. Maternal aging and chromosomal abnormalities: new data drawn from in vitro unfertilized human oocytes. Hum Genet 2003;112(2):195-203

5 American College of Obstetricians and Gynecologists. ACOG practice bulletin. Management of recurrent pregnancy loss. Number 24, February 2001. (Replaces Technical Bulletin Number 212, September 1995). Int J Gynaecol Obstet 2002;78(2):179-190

6 Practice Committee of the American Society for Reproductive Medicine. Evaluation and treatment of recurrent pregnancy loss: a committee opinion. Fertil Steril 2012;98(5):1103-1111

7 National Collaborating Centre for Women's and Children's Health (UK). Ectopic Pregnancy and Miscarriage: Diagnosis and Initial Management in Early Pregnancy of Ectopic Pregnancy and Miscarriage. London: Royal College of Obstetrics and Gynecology; 2012 December (NICE Clinical Guidelines, No. 154)

8 Jaslow CR, Carney JL, Kutteh WH. Diagnostic factors identified in 1020 women with two versus three or more recurrent pregnancy losses. Fertil Steril 2010;93(4):1234-1243

9 Christiansen OB. Evidence-based investigations and treatments of recurrent pregnancy loss. Curr Opin Obstet Gynecol 2006;18(3): 304-312 
10 Jauniaux E, Farquharson RG, Christiansen OB, Exalto N. Evidencebased guidelines for the investigation and medical treatment of recurrent miscarriage. Hum Reprod 2006;21(9):2216-2222

11 Silver RM, Branch DW, Goldenberg R, Iams JD, Klebanoff MA. Nomenclature for pregnancy outcomes: time for a change. Obstet Gynecol 2011;118(6):1402-1408

12 Cunningham FG, MacDonald PC, Gant NF, Leveno KJ, Gilstrap LC. Williams Obstetrics. 19th ed. Norwalk, CT: Appleton and Lange; 1993:661-690

13 Barnhart KT, Sammel MD, Rinaudo PF, Zhou L, Hummel AC, Guo W. Symptomatic patients with an early viable intrauterine pregnancy: HCG curves redefined. Obstet Gynecol 2004;104(1):50-55

14 American College of Obstetricians and Gynecologists Committee on Practice Bulletins-Obstetrics. ACOG Practice Bulletin No. 118: antiphospholipid syndrome. Obstet Gynecol 2011;117(1):192-199

15 American College of Obstetricians and Gynecologists Women's Health Care Physicians. ACOG Practice Bulletin No. 138. Inherited thrombophilias in pregnancy. Obstet Gynecol 2013;122(3):706-717

16 Stephenson MD, Sierra S. Reproductive outcomes in recurrent pregnancy loss associated with a parental carrier of a structural chromosome rearrangement. Hum Reprod 2006;21(4):1076-1082

17 Sugiura-Ogasawara M, Ozaki Y, Sato T, Suzumori N, Suzumori K. Poor prognosis of recurrent aborters with either maternal or paternal reciprocal translocations. Fertil Steril 2004;81(2):367-373

18 Carp H, Guetta E, Dorf H, Soriano D, Barkai G, Schiff E. Embryonic karyotype in recurrent miscarriage with parental karyotypic aberrations. Fertil Steril 2006;85(2):446-450

19 Neri G, Serra A, Campana M, Tedeschi B. Reproductive risks for translocation carriers: cytogenetic study and analysis of pregnancy outcome in 58 families. Am J Med Genet 1983;16(4):535-561

20 Engels H, Eggermann T, Caliebe A, et al. Genetic counseling in Robertsonian translocations $\operatorname{der}(13 ; 14)$ : frequencies of reproductive outcomes and infertility in 101 pedigrees. Am J Med Genet A 2008;146A(20):2611-2616

21 Brigham SA, Conlon C, Farquharson RG. A longitudinal study of pregnancy outcome following idiopathic recurrent miscarriage. Hum Reprod 1999;14(11):2868-2871

22 Ogasawara M, Aoki K, Okada S, Suzumori K. Embryonic karyotype of abortuses in relation to the number of previous miscarriages. Fertil Steril 2000;73(2):300-304

23 Carp H, Toder V, Aviram A, Daniely M, Mashiach S, Barkai G. Karyotype of the abortus in recurrent miscarriage. Fertil Steril 2001;75(4):678-682

24 Hassold TJ. A cytogenetic study of repeated spontaneous abortions. Am J Hum Genet 1980;32(5):723-730

25 Stephenson MD, Awartani KA, Robinson WP. Cytogenetic analysis of miscarriages from couples with recurrent miscarriage: a casecontrol study. Hum Reprod 2002;17(2):446-451
26 Kearns WG, Pen R, Graham J, et al. Preimplantation genetic diagnosis and screening. Semin Reprod Med 2005;23(4):336-347

27 Tobler KJ, Brezina PR, Benner AT, Du L, Xu X, Kearns WG. Two different microarray technologies for preimplantation genetic diagnosis and screening, due to reciprocal translocation imbalances, demonstrate equivalent euploidy and clinical pregnancy rates. J Assist Reprod Genet 2014;31(7):843-850

$28 \mathrm{Li} \mathrm{G}$, Jin H, Xin Z, et al. Increased IVF pregnancy rates after microarray preimplantation genetic diagnosis due to parental translocations. Syst Biol Reprod Med 2014;60(2):119-124

29 Brezina PR, Kutteh WH. Classic and cutting-edge strategies for the management of early pregnancy loss. Obstet Gynecol Clin North Am 2014;41(1):1-18

30 Jaslow CR, Kutteh WH. Effect of prior birth and miscarriage frequency on the prevalence of acquired and congenital uterine anomalies in women with recurrent miscarriage: a cross-sectional study. Fertil Steril 2013;99(7):1916-1922.e1

31 Bailey AP, Jaslow CR, Kutteh WH. Minimally invasive surgical options for congenital and acquired uterine factors associated with recurrent pregnancy loss. Womens Health (Lond Engl) 2015;11(2):161-167

32 Coulam CB, Acacio B. Does immunotherapy for treatment of reproductive failure enhance live births? Am J Reprod Immunol 2012;67(4):296-304

33 Wong LF, Porter TF, Scott JR. Immunotherapy for recurrent miscarriage. Cochrane Database Syst Rev 2014;10:CD000112

34 Egerup P, Lindschou J, Gluud C, Christiansen OB. The effects of immunotherapy with intravenous immunoglobulins versus no intervention, placebo, or usual care in patients with recurrent miscarriages: a protocol for a systematic review with metaanalyses, trial sequential analyses, and individual patient data meta-analyses of randomised clinical trials. Syst Rev 2014;3:89

35 Kutteh WH. Antiphospholipid antibody syndrome and reproduction. Curr Opin Obstet Gynecol 2014;26(4):260-265

36 Salmon JE, Girardi G, Lockshin MD. The antiphospholipid syndrome as a disorder initiated by inflammation: implications for the therapy of pregnant patients. Nat Clin Pract Rheumatol 2007; $3(3): 140-147$, quiz 1,187

37 Je S, Girardi G. The role of complement in the antiphospholipid syndrome. Curr Dir Autoimmun 2004;7:33-48

38 Kutteh WH. Antiphospholipid antibody-associated recurrent pregnancy loss: treatment with heparin and low-dose aspirin is superior to low-dose aspirin alone. Am J Obstet Gynecol 1996; 174(5):1584-1589

39 Lund M, Kamper-Jørgensen M, Nielsen HS, Lidegaard Ø, Andersen AM, Christiansen OB. Prognosis for live birth in women with recurrent miscarriage: what is the best measure of success? Obstet Gynecol 2012;119(1):37-43 